\title{
NOUVELLE
}

\section{La diversité des interactions plantes-pollinisateurs Un pré-requis indispensable à la stabilité des écosystèmes}

Colin Fontaine, Jacques Meriguet, Michel Loreau, Isabelle Dajoz

\section{Fontaine, J. Meriguet, I. Dajoz : UMR CNRS 7618,}

Biogéochimie et écologie des milieux continentaux (BIOEMCO), École Normale Supérieure,

46, rue d'Ulm, 75230 Paris

Cedex 05, France.

colin.fontaine@ens.fr

M. Loreau : Department of Biology, McGill University, Montréal, Québec, Canada. et le fonctionnement des écosystèmes est une des grandes problématiques de l'écologie [1]. L'acquisition de connaissances solides sur ce point devient cruciale dans le contexte actuel de crise de la biodiversité. Les pertes d'espèces liées directement ou indirectement aux activités humaines se produisent à un rythme 100 à 1000 fois supérieur aux extinctions naturelles, et il devient indispensable de connaître l'impact de cette perte de biodiversité sur le fonctionnement et la capacité de régénération des écosystèmes [2].

L'intégrité fonctionnelle des écosystèmes est primordiale: de très nombreux services sont rendus aux sociétés humaines via le bon fonctionnement des écosystèmes comme par exemple l'épuration des eaux ou le maintien de la fertilité des sols [3]. Ils passent souvent inaperçus et sont pourtant indispensables. La pollinisation des plantes à fleurs par les animaux fait également partie de ces services écosystémiques [4]. En effet, plus de $70 \%$ des espèces de plantes supérieures sont visitées par les animaux pollinisateurs, qui assurent leur reproduction et donc leur survie [5]. Les plantes, en tant que producteurs primaires, sont les organismes qui vont capter l'énergie du rayonnement solaire et fixer le $\mathrm{CO}_{2}$ atmosphérique pour former de la matière organique. Elles sont donc à la base des flux de matière et d'énergie réalisés au sein des écosystèmes.

L'impact de la diversité des animaux pollinisateurs sur le succès reproducteur des plantes supérieures commence à être connu à l'échelle d'une espèce de plante $[6,7]$, mais qu'en est-il à l'échelle des communautés végétales? La réponse à cette question n'est pas triviale. En effet, à ce niveau, les relations entre plantes et pollinisateurs forment des réseaux d'interactions complexes [8] où se côtoient des espèces totalement généralistes, totalement spécialistes ainsi que tous les intermédiaires possibles [9].

Il est donc urgent de mieux connaître l'impact de la perte de biodiversité sur les réseaux d'interactions plurispécifiques [10]. L'approche expérimentale présentée ici y contribue [11]; elle vise à tester l'impact de la diversité sur la stabilité des réseaux d'interactions plantes-pollinisateurs en suivant une approche fonctionnelle.

\section{Groupes fonctionnels \\ analysés sur deux années}

Des groupes fonctionnels de plantes et d'insectes pollinisateurs ont été construits, sur la base de caractères morphologiques:

- pour les plantes, nous avons considéré l'accessibilité des récompenses offertes aux pollinisateurs (pollen et nectar). Ces dernières pouvaient être soit faciles d'accès (trois espèces avec des corolles plates, en forme d'《assiette »), soit difficiles (trois espèces avec des corolles profondes, en «tube »);

- pour les pollinisateurs, nous avons considéré la longueur des pièces buccales: soit courtes (trois espèces de diptères), soit longues (trois espèces d'hyménoptères)
Un réseau théorique d'interactions peut être bâti avec ces quatre groupes fonctionnels (Tableau I). En raison des contraintes morphologiques, les espèces spécialistes (de plantes ou bien d'insectes) devraient interagir préférentiellement avec les espèces généralistes (d'insectes ou de plantes).

Nous avons créé des communautés végétales expérimentales, en faisant varier la diversité fonctionnelle des plantes. Au total, il y avait trois types de communautés végétales, contenant soit l'un, soit l'autre, soit les deux groupes fonctionnels de plantes, à densité contrôlée. Toutes les communautés (36 en tout) ont été recouvertes de cages cubiques en voile de nylon afin d'en contrôler la pollinisation (Figures

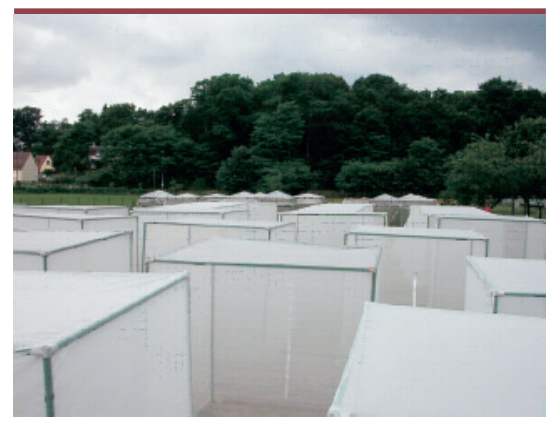

Figure 1. Vue générale du dispositif utilisé sur le terrain, avec les cages à pollinisation en voile de nylon contenant les communautés expérimentales de plantes et de pollinisateurs. 
$I$ et 2). Chaque type de communauté a subi 3 traitements de pollinisation, soit par les pollinisateurs à pièces buccales longues, ou à pièces buccales courtes, soit par les deux groupes fonctionnels d'insectes ensemble. L'expérience a été réalisée pendant 2 années consécutives, en contrôlant le nombre total de visites des insectes sur les plantes. Nous avons ensuite analysé le succès reproducteur (production de fruits et de graines) et le recrutement (nombre de plantules qui poussent après deux ans de traitement) des différentes espèces dans chaque communauté végétale. Nous avons pu ainsi répertorier le nombre d'espèces qui se reproduisaient et se maintenaient dans chaque type de communauté, après 2 ans d'expérimentation.

\section{Résultats}

Nous avons constaté que l'impact des traitements de pollinisation sur le succès reproducteur des plantes n'est pas le même dans les trois communautés végétales expérimentales. Dans les communautés composées uniquement du groupe fonctionnel «fleurs en assiette », le succès reproducteur est identique, quelle que soit la diversité des pollinisateurs. $\varepsilon$ n revanche, dans les

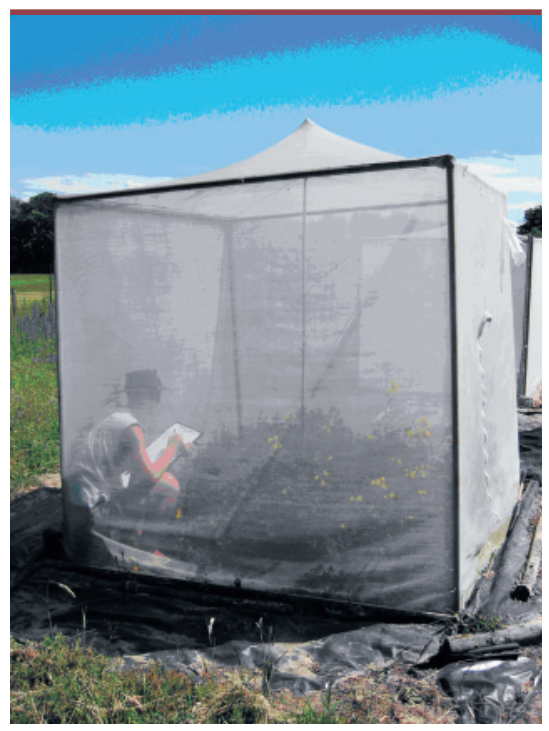

Figure 2. Séance d'observation du comportement de butinage. communautés composées uniquement du groupe fonctionnel «fleurs en tube », le succès reproducteur est significativement plus élevé quand les plantes sont butinées par des pollinisateurs à pièces buccales longues. Enfin, ce sont dans les communautés avec la plus haute diversité fonctionnelle, aussi bien en termes de plantes que de pollinisateurs que la production de graines et de fruits est la plus importante.

De plus, nos résultats montrent que la manipulation de la diversité fonctionnelle des pollinisateurs a des effets à long terme sur la stabilité de nos communautés végétales expérimentales (Figure 3). La diversité des pollinisateurs n'a pas eu d'effets sur le recrutement de la communauté végétale composée uniquement du groupe fonctionnel «fleurs en assiette», comme le prédit le diagramme d'interactions théoriques du Tableau I. En revanche, dans la communauté «fleurs en tube », le recrutement est le moins bon avec un butinage effectué uniquement par des insectes à pièces buccales courtes, ce qui est également en accord avec nos prédictions. De façon plus surprenante, un effet positif très fort de la diversité fonctionnelle des pollinisateurs émerge dans la communauté végétale contenant les deux groupes fonctionnels de plantes. En d'autres termes, la diversité fonctionnelle des pollinisateurs influence d'autant plus la stabilité de la communauté végétale que la diversité de cette dernière est importante. Quel est le mécanisme à la base de cet effet de la diversité? Nous avons analysé précisément la répartition des visites des pollinisateurs sur chaque espèce de plante, dans chaque traitement de pollinisation. Dans des conditions de diversité fonctionnelle maximale, chaque espèce d'insecte se focalise sur la(les) plante(s) dont il est le pollinisateur le plus efficace: il retire un maximum de récompenses en un minimum de temps. Cette efficacité accrue de butinage accroît le succès reproducteur de chaque espèce de plante, et influe donc sur le maintien à long terme de la communauté végétale.

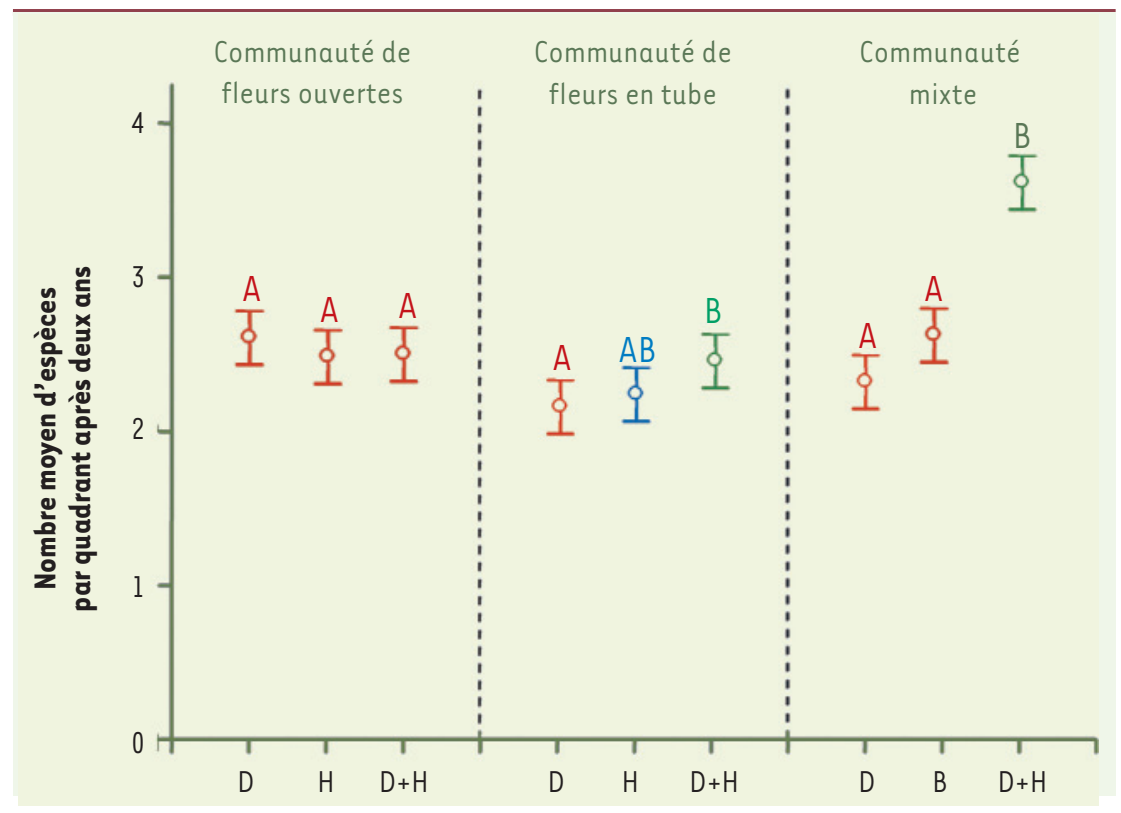

Figure 3. Effets des différents traitements de pollinisation sur le recrutement des communautés végétales expérimentales. Nombre moyen d'espèces par quadrant de $0,25 \mathrm{~m}^{2}$ dans les trois types de communautés végétales, pollinisées par les diptères $(D)$, les hyménoptères $(H)$, ou les deux groupes de pollinisateurs $(D+H)$. Les barres d'erreur représentent une erreur standard, les moyennes portant la même lettre sont non significativement différentes au seuil de $p=0,05$. 


\begin{tabular}{|c|c|c|c|c|}
\hline $\begin{array}{c}\text { Espèces } \\
\text { et groupes } \\
\text { fonctionnels } \\
\text { de pollinisateurs }\end{array}$ & $\begin{array}{c}\text { Longueur } \\
\text { moyenne des } \\
\text { pièces buccales } \\
\text { (mm } \pm \text { écart-type) }\end{array}$ & Réseau de pollinisation théorique & $\begin{array}{c}\text { Accessibilité } \\
\text { des récompenses } \\
\text { offertes } \\
\text { aux pollinisateurs }\end{array}$ & $\begin{array}{c}\text { Espèces } \\
\text { et groupes } \\
\text { fonctionnels } \\
\text { de plantes }\end{array}$ \\
\hline Saephoria sp. & $2,66 \pm 0,35$ & & Facile & Matricaria officinalis \\
\hline Eristalis balteatus & $2,3 \pm 0,20$ & & Facile & Erodium cicutarium \\
\hline Eristalis tenax & $5,47 \pm 0,29$ & Diph & Facile & $\begin{array}{l}\text { Raphanus } \\
\text { raphanistrum }\end{array}$ \\
\hline Bombus terrestris & $9,02 \pm 0,19$ & & Difficile & Mimulus guttatus \\
\hline $\begin{array}{c}\text { Bombus } \\
\text { pascuorum }\end{array}$ & $9,21 \pm 1,02$ & & Difficile & Medicago sativa \\
\hline $\begin{array}{l}\text { Bombus } \\
\text { lapidarius }\end{array}$ & $8,10 \pm 0,86$ & Hyménoptères & Difficile & Lotus corniculatus \\
\hline
\end{tabular}

Tableau I. Structure du réseau de pollinisation expérimental, espèces utilisées dans chaque groupe fonctionnel d'insectes (à gauche) et de plantes (à droite). Les caractéristiques sur lesquelles sont bâties chaque groupe fonctionnel (taille des pièces buccales, accessibilité des ressources) sont indiquées.

\section{Conclusions}

Nos résultats montrent que, après 2 ans, les communautés végétales pollinisées par la plus grande diversité fonctionnelle d'insectes contiennent environ $50 \%$ d'espèces de plantes en plus que celles pollinisées par une diversité fonctionnelle moindre. Certes, ces résultats expérimentaux ont été obtenus sur des communautés très simplifiées de plantes et de pollinisateurs, mais ce pré-requis est indispensable à toute démarche expérimentale en conditions contrôlées. Par ailleurs, toutes les communautés naturelles de plantes contenant une grande diversité de morphologies florales, nous pouvons supposer que la diversité fonctionnelle des pollinisateurs jouera un rôle très important dans la stabilité à long terme des communautés végétales naturelles.
En conclusion, ces résultats démontrent l'importance cruciale de la diversité des pollinisateurs dans la stabilité des communautés végétales. Très vraisemblablement, l'extinction de pollinisateurs aurait pour conséquence l'extinction d'espèces végétales. Étant donné l'importance de la diversité des espèces végétales dans les processus écosystémiques (les plantes, producteurs primaires, sont le niveau trophique de base de l'écosystème, dont dépendent plus ou moins directement tous les autres niveaux trophiques), nos résultats indiquent que la diversité fonctionnelle des réseaux de pollinisation est indispensable au fonctionnement et à la stabilité de la biosphère. $\diamond$

Diversity of plant-pollinator

interaction and stability of ecosystems

\section{RÉFÉRENCES}

1. Loreau M, Naeem S, Inchausti P, et al. Biodiversity and ecosystem functioning: current knowledge and future challenges. Science $2001 ; 294: 804-8$.
2. Naeem S, Thompson LJ, Lawler SP, et al. Declining biodiversity can alter the performance of ecosystems. Nature 1994 ; 368 : $734-7$

3. Costanza R, Arge R, deGroot R, et al. The value of the world's ecosystem services and natural capital. Nature 1997 ; $387: 253-60$.

4. Kearns CA, Inouye DW, Waser NM. Endangered mutualisms: the conservation of plant-pollinator interactions. Annu Rev Ecol System 1998 ; 29 : 83-112.

5. Axelrod DI. The evolution of flowering plants. In: Tax $S$, ed. Evolution after Darwin, vol. 1. The evolution of life. Chicago: University of Chicago Press, $1960: 227-305$.

6. Kremen C, Williams NM, Thorp RW. Crop pollination from native bees at risk from agricultural intensification. Proc Natl Acad Sci USA 2002 ; 99: 16812-6.

7. Klein AM, Steffan-Dewenter I, Tscharntke T. Fruit set of highland coffee increases with the diversity of pollinating bees. Proc Roy Soc Lond B 2003 ; $270: 955-61$.

8. Memmott J. The structure of a plant-pollinator food web. Ecol Lett $1999 ; 2$ : 276-80.

9. Bascompte J, Jordano P, Melian CJ, et al. The nested assembly of plant-animal mutualistic networks. Proc Natl Acad Sci USA 2003 ; 100: 9383-7.

10. Cardinale BJ, Palmer MA, Collins SL. Species diversity enhances ecosystem functioning through interspecific facilitation. Nature $2002 ; 415$ : 426-9.

11. Fontaine C, Dajoz I, Meriguet J, Loreau M. Functional diversity of plant-pollinator interaction webs enhances the persistence of plant communities. PLOS Biol $2006 ; 4: 129-35$.

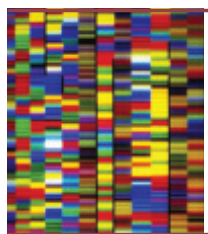

Retrouvez chaque mois médecine/sciences sur France-Info dans la chronique « Info-Sciences 》 de Marie-Odile Monchicourt, du lundi au mercredi. france-info.com 